\title{
Luminescent Chemosensor Systems for Detecting Metal Ions in Aqueous Media
}

\author{
Andrey A. Leonov ${ }^{1 *}$, Alexander A. Sergeev ${ }^{1}$, Alexander Yu. Mironenko ${ }^{2}$, \\ and Sergey S. Voznesenskiy ${ }^{1}$ \\ ${ }^{1}$ Institute of Automation and Control Processes, Far Eastern Branch of the Russian Academy of Sciences, 5 Radio Str., \\ Vladivostok 690041, Russia \\ ${ }^{2}$ Institute of Chemistry, Far Eastern Branch of the Russian Academy of Sciences, 159 Prosp. 100-letiya Vladivostoka, \\ Vladivostok 690022, Russia
}

* e-mail: andreileonov@inbox.ru

\begin{abstract}
The paper deals with the synthesis and study of chemosensor structures for detecting metal ions in aqueous solutions based on a hydrophilic polymer modified by an ion-sensitive indicator. A new ion-sensitive indicator based on the rhodamine 6G luminophore that selectively responds to the presence of nickel ions in aqueous environment is developed. The limit of the nickel ions detection is equal to $0.1 \mu \mathrm{M}$. (C) 2018 Journal of Biomedical Photonics \& Engineering.
\end{abstract}

Keywords: biopolymer; chitosan; optical sensor; sensitive coatings; luminescence; analyte; polymers; metal ions.

Paper \#3277 received 15 Jan 2018; revised manuscript received 24 Feb 2018; accepted for publication 26 Feb 2018; published online 20 Mar 2018. doi: 10.18287/JBPE18.04.010502. [Special Section. Workshop "Biophotonics" of the XV all-Russian Youth Samara conference-contest on optics and laser physics].

\section{References}

1. L. Prodi, F. Bolletta, M. Montalti, and N. Zaccheroni, "Luminescent chemosensors for transition metal ions," Coordination Chemistry Reviews 205(1), 59-83 (2000).

2. Y. Xiao, Y. Cui, Q. Zheng, S. Xiang, G. Qian, and B. Chen, "A microporous luminescent metal-organic framework for highly selective and sensitive sensing of $\mathrm{Cu} 2+$ in aqueous solution," Chemical Communications 46(30), 5503-5505 (2010).

3. L. Prodi, "Luminescent chemosensors: from molecules to nanoparticles," New Journal of Chemistry 29(1), 456-461 (2005).

4. M. Formica, V. Fusi, L. Giorgi, and M. Micheloni, "New fluorescent chemosensors for metal ions in solution," Coordination Chemistry Reviews 256(1-2), 170-192 (2012).

5. F. M. Raymo, and I. Yildiz, "Luminescent chemosensors based on semiconductor quantum dots," Physical Chemistry Chemical Physics 9(17), 2036-2043 (2007).

6. K. Lunstroot, K. Driesen, P. Nockemann, C. Görller-Walrand, K. Binnemans, S. Bellayer, J. Le Bideau, and A. Vioux, "Luminescent Ionogels Based on Europium-Doped Ionic Liquids Confined within Silica-Derived Networks," Chemistry of Materials 18(24), 5711-5715 (2006).

7. A. Prasanna de Silva, T. S. Moodyb, and G. D. Wright, "Fluorescent PET (Photoinduced Electron Transfer) sensors as potent analytical tools," Analyst 134(12), 2385-2393 (2009).

8. R. M. Clegg, "Fluorescence resonance energy transfer and nucleic acids," Methods in Enzymology 211, 353388 (1992).

9. Y. Jeong, and J. Yoon, "Recent progress on fluorescent chemosensors for metal ions," Inorganica Chimica Acta 381(23), 2-14 (2012).

10. S. Bonacchi, D. Genovese, R. Juris, M. Montalti, L. Prodi, E. Rampazzo, M. Sgarzi, and N. Zaccheroni, "Luminescent Chemosensors Based on Silica Nanoparticles," in Topics in Current Chemistry, vol. 300, L. Prodi, M. Montalti, N. Zaccheroni (Eds), Springer, Berlin, Heidelberg, 93-138 (2010).

11. N. R. Chereddy, K. Suman, P. S. Korrapati, S. Thennarasu, and A. B. Mandal, "Design and synthesis of rhodamine based chemosensors for the detection of $\mathrm{Fe}^{3+}$ ions," Dyes and Pigments 95(3), 606-613 (2012). 
12. J. Y. Kwon, Y. J. Jang, Y. J. Lee, K. M. Kim, M. S. Seo, W. Nam, and J. Yoonet, “A Highly Selective Fluorescent Chemosensor for $\mathrm{Pb}^{2+}$," American Chemical Society 127(28), 10107-10111 (2005).

13. Y. Zhou, J. Zhang, L. Zhang, Q. Zhang, T. Ma, and J. Niu, "A rhodamine-based fluorescent enhancement chemosensor for the detection of $\mathrm{Cr}^{3+}$ in aqueous media," Dyes and Pigments 97(1), 148-154 (2013).

14. Y. K. Jang, U. C. Nam, H. L. Kwon, I. H. Hwang, and C. Kim, "A selective colorimetric and fluorescent chemosensor based-on naphthol for detection of $\mathrm{Al}^{3+}$ and $\mathrm{Cu}^{2+}$," Dyes and Pigments 99(1), 6-13 (2013).

15. Z.-C. Liao, Z.-Y. Yang, Y. Li, B.-D. Wang, and Q.-X. Zhou "A simple structure fluorescent chemosensor for high selectivity and sensitivity of aluminum ions," Dyes and Pigments 97(1), 124-128 (2013).

16. M. R. Ganjali, M. Hosseini, M. Motalebi, M. Sedaghat, F. Mizani, F. Faridbod, and P. Norouzi, "Selective recognition of $\mathrm{Ni} 2+$ ion based on fluorescence enhancement chemosensor," Spectrochimica Acta Part A: Molecular and Biomolecular Spectroscopy 140, 283-287 (2015).

17. A. Yari, M. B. Gholivand, and F. Rahhedayat, "Development and characterization of a new nickel(II) ion selective optode based on 2-amino-1-cyclopentenedithiocarboxylic acid," Measurement 44(9), 1691-1698 (2011).

18. N. Aksuner, E. Henden, I. Yilmaz, and A. Cukurovali, "A novel optical chemical sensor for the determination of nickel(II) based on fluorescence quenching of newly synthesized thiazolo-triazol derivative and application to real samples," Sensors and Actuators B: Chemical 166-167, 269-274 (2012).

19. H. Li, S.-J. Zhang, C.-L. Gong, Y.-F. Li, Y. Liang, Z.-G. Qi, and S. Chen, "Highly sensitive and selective fluorescent chemosensor for Ni2+ based on a new poly(arylene ether) with terpyridine substituent groups," The Analyst 138(23), 7090-7093 (2013).

\section{Introduction}

At present, the optical measurement methods allow efficient registration of different heavy metals ions in aqueous solutions $[1,2]$. This is due to a number of advantages possessed by these methods, such as short response time, good reproducibility, wide range of determined concentrations, and high resistance to noise electromagnetic fields. One of the widely used ways to organise such sensor systems is to use luminescent indicators [3], which can change their luminescence properties in the presence of the measured substance (analyte). These indicators can be obtained from different chemical compounds, e.g., dyes [4], quantum dots [5], and rare-earth metals [6].

As a rule, the sensor response of such systems is based on the effects of photoinduced electron transfer (PET) and fluorescence resonance transfer (FRET) of the excitation energy. These effects are based on the energy exchange between chemosensitive part of indicator (donor) to luminescence centre (acceptor) that causes the change of acceptor luminescence properties. In dependence of transfer kind, the energy transfer occurs in an own specific way. In the case of PET, after the molecule excitation, the electron is transferred from the highest occupied molecular orbital to the lowest unoccupied molecular orbital, giving rise to luminescence intensity [7]. In the case of FRET, the energy transfer from donor to acceptor can occur without the emission of photons and due to dipoledipole interactions between the donor and the acceptor, which causes the intramolecular charge transfer [8].

In the case of FRET-based sensors, it is possible to detect the ions $\mathrm{Zn}^{2+}, \mathrm{Cu}^{2+}, \mathrm{Al}^{3+}, \mathrm{Cd}^{2+}$ in an aqueous solution using different luminescent indicators based on pyrene, coumarin, anthracene, etc. $[9,10]$. To detect the $\mathrm{Fe}^{3+}$ ions can be used the PET-effect in the rhodamine B luminophore, with achieved detection limit equal to
$10^{-6} \mathrm{M} / 1$ [11]. Using the same luminophore, one can detect the $\mathrm{Pb}^{2+}$ ions in the acetonitrile medium [12], and the $\mathrm{Cr}^{3+}$ ions in the aqueous solution with the detection limit $10^{-3} \mathrm{M} / 1$ [13].

Basing on the above methods it is possible to obtain chemosensor systems possessing good selectivity, e.g., using the indicator based on the hydroxynaphthalene (naphthol), one can detect $\mathrm{Al}^{3+}$ ions with sufficient accuracy even in the presence of other metal ions [14]. The other one chemosensor based on the methyl pyrazinylketone benzoyl hydrazone (MPBH) indicator is able to detect the $\mathrm{Al}^{3+}$ ions with the concentration as low as $10^{-7} \mathrm{M} / 1[15]$.

At the same time, the determination of metal ion concentrations in aqueous medium by direct use of luminescent indicators in most cases is limited to laboratory conditions studies and is hardly adaptable to the measurements in real environment. Thus, the development of new sensor system that will combine the sensitivity of analytical methods with the possibility to perform measurements under the real conditions is very important.

Here, we report the synthesis and optical and sensor characteristics studies of a new ion-sensitive indicator based on rhodamine 6G. A sensitive element for the luminescent detectors of nickel ions in aqueous solutions based on modification of a thin hydrophilic polymer film with the obtained indicator was developed.

To date there are a very few publications on the development of optical sensors for direct detection of nickel in aqueous media. The detection of nickel ions is mainly implemented in buffer solutions of organic compounds (acetonitrile or dimethylformamide), which are enhancing the efficiency of the analyte ion chelation by the chemosensitive receptor. Thus, the authors of Ref. [16] use the chemosensor based on 2-(1-H-benzo[d]imidazol-2yl)-N-phenyl hydrazine 
carbothioamide to detect nickel ions in the buffer solution of acetonitrile with the detection limit of $7.9 \times 10^{-8} \mathrm{M}$. The method proposed in [16] is simple, possesses good sensitivity and selectivity. Although the studies were carried out in buffer solution, the authors declare the possibility of using this sensor for detecting $\mathrm{Ni}^{2+}$ ions in wastewater samples. In Ref. [17], the 2amino-1-cyclopentene-dithiocarboxylic acid formed the sensitive element of the chemosensor intended for nickel ion detection; the range of determined concentration was from $3.1 \times 10^{-8}$ to $8.0 \times 10^{-3} \mathrm{M}$. In Ref [18] was developed the chemosensor based on the 2-\{6-(3-methyl-3-mesitylcyclobutyl)-thiazolo[3,2-

b] $[1,2,4]$ triazol-2-yl $\}$-phenol with the range of determined concentrations from $1.0 \times 10^{-9}$ to $4.4 \times 10^{-3} \mathrm{M}$. Both in Ref. [17] and in Ref. [18] the studies of the sensor response were performed in the acetonitrile buffer. In Ref. [19] sensing studies for nickel detection were performed in dimethylformamide buffer solution, obtained limit of detection was equal to $0.01 \mu \mathrm{M} / 1$.

In contrast to the papers mentioned above, here we perform sensing measurements in simple sodium acetate buffer making no significant influence on the metal ions chelation process and serving only for stabilising the working solution $\mathrm{pH}$ level.

\section{Materials and methods}

Fig. 1 illustrates the process of the rhodamine $6 \mathrm{G}$ ionsensitive derivatives synthesis, which consists in the replacement of the functional $\mathrm{O}-\mathrm{CH}_{3}$ group with a nitrogen-containing ligand. The bonding of this group (N-ligand, Fig. 1) leads to the occurrence of photoinduced electron transfer, that quench the luminescence of the synthesised compound. However, in the case of chelating the metal ion by this group, the photoinduced transfer of electron does not occur, and the synthesised complex becomes luminescent.

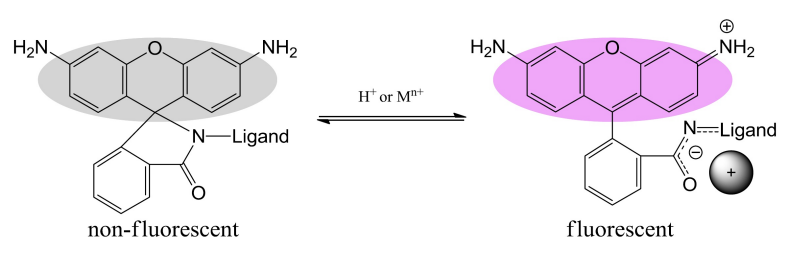

Fig. 1 The general principle of the sensor response formation by the synthesised indicator.

The synthesis of ion-sensitive indicator was performed in two steps. At the first step, we obtained the rhodamine $6 \mathrm{G}$ lactam. At the second stage rhodamine $6 \mathrm{G}$ lactam was modified with acetylacetone ligand.

To form the high-sensitivity sensor structures, we used $1 \%$ aqueous solution of high-molecular chitosan (Sigma) (the deacetylation degree $80 \%$, the molecular mass $\approx 10^{6} \mathrm{Da}$ ) in $1 \%$ acetic acid. The coatings were deposited on glass substrates with the dimensions $2.5 \times 2.5 \mathrm{~cm}$ by solution spin-coating on the Laurell WS400B-6NPP-LITE device at the angular velocities 1000 revolutions per minute. The coating thickness measured by optical reflectometry technique (on Sentech SE500adv) was about $500 \mathrm{~nm}$. After the deposition, chitosan coatings were annealed during 10 minutes at $150{ }^{\circ} \mathrm{C}$, deprotonated in the $3 \%$ ammonia solution, and dried in air. For doping with the indicator, the coatings were immersed in the solution with the component content $0.05 \%$ for 30 minutes. After the sorption, the substrates were carefully washed with deionised water and dried in air.

To study the sensor characteristics of the obtained ion To study the sensor characteristics of the obtained ion-sensitive indicator, we prepared aqueous solutions of salts of different two- and three-valence metals with the concentration $1 \pm 0.2 \mu \mathrm{M}$. Since the metal salts dissipation in the aqueous medium increased its acidity (lowering the $\mathrm{pH}$ level), the measurements in distilled water is related to essential increase of the measurement error. To equalise the experimental conditions in the studies of the sensor response for different metal ions, we prepared the sodium acetate buffer with the $\mathrm{pH} \approx 5.6$. The sodium acetate buffer $(0.2 \mathrm{M})$ was prepared by mixing $91 \mathrm{ml}$ of the sodium acetate aqueous solution (with the concentration of $\mathrm{NaOAc} 0.2$ $\mathrm{M})$ and $9 \mathrm{ml}$ of the acetic acid aqueous solution with the similar concentration.

The absorption spectra were recorded using spectrophotometer Varian Cary 5000i with $2 \mathrm{~nm}$ scanning step and $0.1 \mathrm{~s}$ averaging time. The sample was placed perpendicular to the incident light beam.

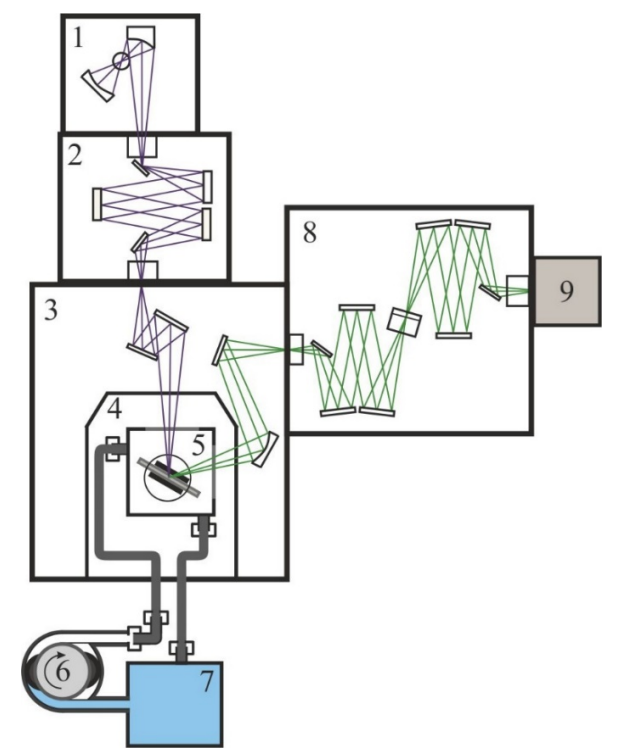

Fig. 2 Block diagram of the spectrofluorimeter Horiba Fluorolog 3 with the designed measurement chamber: 1 - radiation source unit, 2 - exciting radiation monochromator, 3 - unit of focusing the exciting radiation and the luminescence, 4 - measurement section, 5 - hermetic chamber with the fixed sample, 6 - peristaltic pump, 7 - vessel with the analysed aqueous solution, 8 - emission monochromator, 9 detector. 
The fluorescence spectra were recorded using spectrofluorimeter Horiba Fluorolog 3 with $1 \mathrm{~nm}$ step and $0.1 \mathrm{~s}$ averaging time. The sample was placed at $45^{\circ}$ with the exciting radiation beam to provide the maximal signal level.

To perform the measurements under the conditions close to the real ones, we designed and constructed a sealed chamber compatible with the measurement unit of the spectrofluorimeter (Fig. 2). As a radiation source (1) we used the continuous-wave broadband $450 \mathrm{~W}$ xenon lamp.

\section{Results and discussion}

Fig. 3 presents the absorption spectra of the initial rhodamine $6 \mathrm{G}$ and ion-sensitive indicator based on it. One can see that after the modification the optical density (directly proportional to the absorption coefficient of substance) and the intensity of luminescence are reduced, which is an evidence of photoinduced transfer of electron from the base part of the rhodamine molecule to the ligand. Rhodamine $6 \mathrm{G}$ lactam demonstrates the domination of the absorption peak near $500 \mathrm{~nm}$ compared to initial rhodamine peak at $532 \mathrm{~nm}$. Moreover, the rhodamine $6 \mathrm{G}$ lactam luminescence spectrum is red-shifted towards by at least $30 \mathrm{~nm}$.

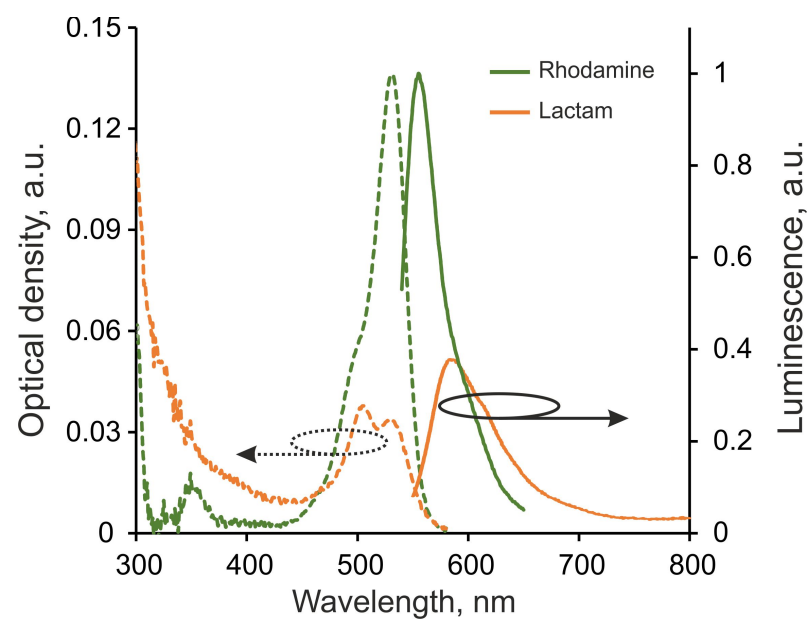

Fig. 3 Spectral characteristics of rhodamine $6 \mathrm{G}$ and the ion-sensitive indicator based on it. The dashed and solid lines correspond to the absorption and luminescence, respectively.

Fig. 4 shows the rhodamine $6 \mathrm{G}$ lactam luminescence spectra in the presence of various metal ions. One can see that the maximal luminescence intensity increase is observed for nickel ions. Some increase of the luminescence intensity occurs also in the presence of copper and silver, but in this case the luminescence intensity increase is much weaker. This might be due to formation of weak chemical bond between acetylacetone and silver and copper ions which has much lower stability compared to the acetylacetonenickel complex.

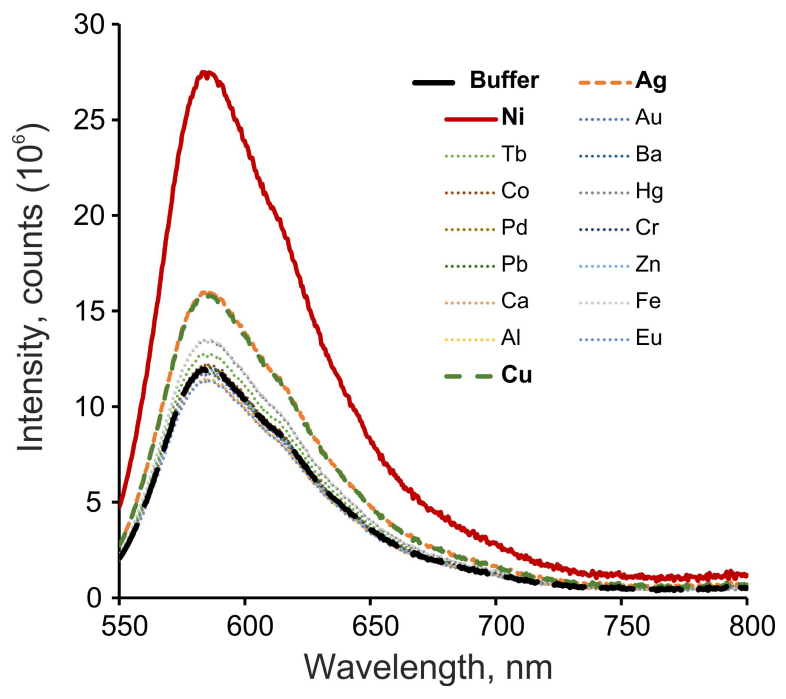

Fig. 4 Intensity of luminescence of rhodamine 6G lactam affected by different metal ions.

The rhodamine $6 \mathrm{G}$ lactam luminescence intensity enhancement is proportional to the analyte concentration (Fig. 5). But, one can notice some specific features of the sensor response formation. In the initial state, the rhodamine $6 \mathrm{G}$ lactam luminescence is essentially lower than the intensity of luminescence of rhodamine $6 \mathrm{G}$ (Fig. 3). The sensor response of the rhodamine $6 \mathrm{G}$ lactam is characterised by practically linear growth of the luminescence intensity under the increase of the analyte concentration. This is confirmed by the logarithmic approximation of the dependence of the luminescence intensity on the logarithm of the analyte concentration with the coefficient of determination $\left(\mathrm{R}^{2}\right)$ close to unity (Fig. 5(b)). At the same time, the increase of the analyte concentration leads to some changes in the luminescence spectrum of the indicator. Thus, at the analyte concentrations above $1 \mu \mathrm{M}$ the maximum of luminescence demonstrates $\Delta \lambda \approx 5 \mathrm{~nm}$ blue-shift. Such behaviour can be due to exceeding the ratio $1: 1$ for the chelated metal ions to chelating groups.

In other words, at low concentrations of metal ions each chemosensitive receptor binds only one analyte ion. Increasing of nickel ions concentration leads to the binding of an additional analyte ion by the chelating centre, which results in occurrence of additional changes of the electron transfer in the indicator molecule. An evidence of this process is also the fact that even at high analyte concentrations $(100 \mu \mathrm{M}$ and higher) no saturation of the sensor response was observed.

The studies of the kinetics of sensor response formation were carried out with the analyte concentration equal to $1 \mu \mathrm{M}$ (the maximum allowable concentration for nickel amounts to $1.7 \mu \mathrm{M}$ in the drinking water). Luminescence spectra were recorded every 15 minutes until the luminescence saturation had been achieved. Between the measurements, the studied solution was kept in darkness in order to avoid the possible photodegradation of the luminophore. 


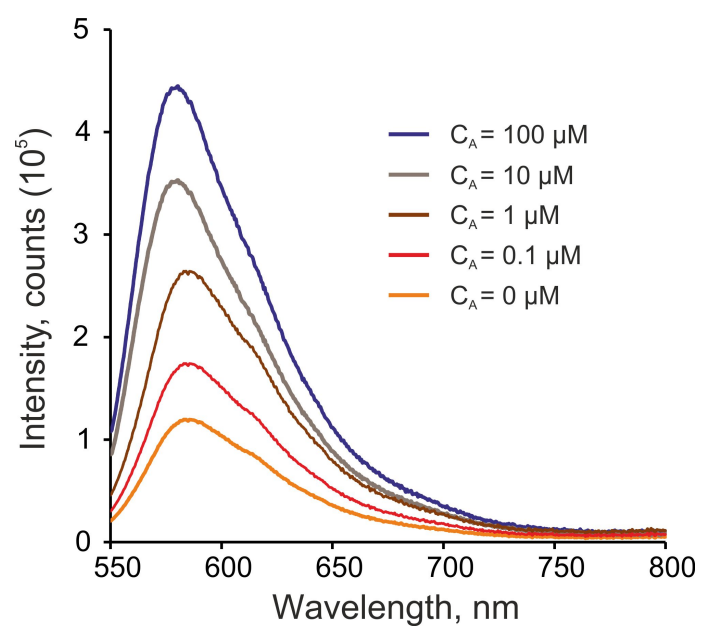

(a)

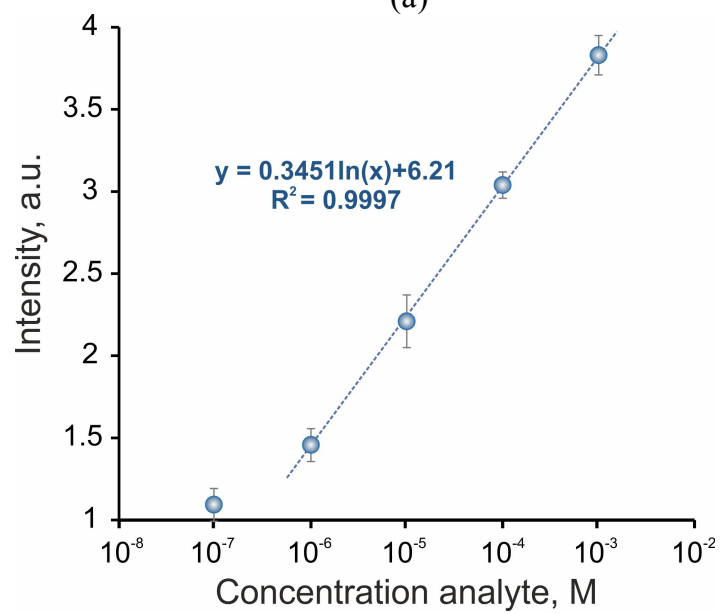

(b)

Fig. 5 Sensor characteristics of rhodamine 6G lactam for different analyte concentrations: the luminescence spectra (a) and the sensor response (b).

From the data presented in Fig. 6, one can see that the time of the sensor response formation for rhodamine 6G lactam amounts to about 110 minutes. During this time one can observe sufficiently strong deviations of the luminescence intensity function from the linear dependence (the value of $\mathrm{R}^{2}$ for the approximation by a linear function differs from unity rather strongly). This effect is, apparently, one more evidence of the process of binding of several analyte ions by one chelating centre.

Alongside with the change of luminescence characteristics during sensor response formation, the changes of the absorption spectra also occur. Thus, increasing of optical absorption at the wavelength of rhodamine $6 \mathrm{G}$ excitation $(\lambda \approx 532 \mathrm{~nm})$ under the increase of the analyte concentration (Fig. 7) is clearly observable. Considering this fact, we can conclude that the luminescence intensity is increasing during the sensor response formation occurs due to greater excitation energy absorption directly by the luminescing part of the rhodamine $6 \mathrm{G}$ lactam. This confirms that sensor response formation related to electron transfer changes in the ion-sensitive indicator molecule during the process of analyte chelation.

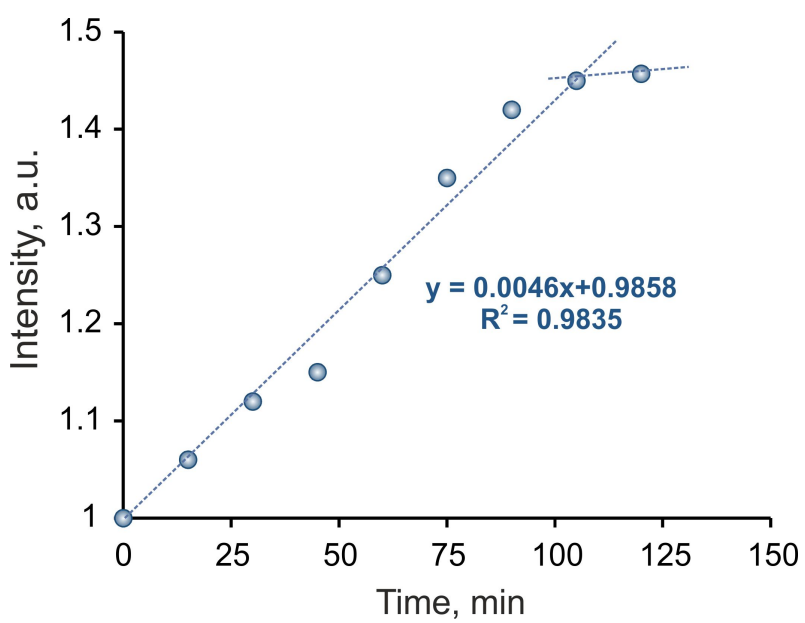

Fig. 6 Time dependence of the luminescence intensity in the rhodamine $6 \mathrm{G}$ derivative affected by $1 \mu \mathrm{M}$ analyte.

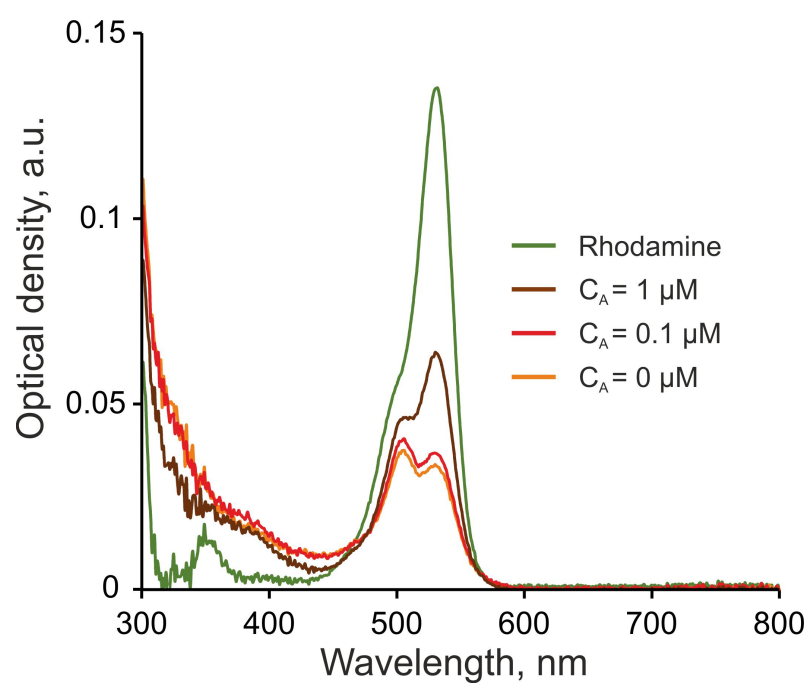

Fig. 7 Optical absorption of the rhodamine 6G lactam affected by different analyte concentrations.

At low analyte concentrations (of the order of $0.1 \mu \mathrm{M}$ ) the uniform increase of the absorption occurs while the initial spectral features of rhodamine $6 \mathrm{G}$ absorption (dominance of the absorption band at $\lambda \approx 500$ $\mathrm{nm}$ over band at $\lambda \approx 532 \mathrm{~nm}$ ) remains unchanged. For the analyte concentrations above $1 \mu \mathrm{M}$, the absorption at $\lambda \approx 532 \mathrm{~nm}$ become higher than at $\lambda \approx 500 \mathrm{~nm}$. That change can determine the rhodamine $6 \mathrm{G}$ lactam luminescence spectral changes at high analyte concentrations (Fig. 5 (a)).

\section{Conclusion}

A new ion-sensitive indicator based on rhodamine $6 \mathrm{G}$ chemical modification was obtained. This indicator selectively responds to the presence of nickel ions in the aqueous environment. The sensor response formation occurs due to changes of electronic transfer in rhodamine-derivate molecule under the interaction with nickel ions. The detection limit for the nickel ions is equal to $0.1 \mu \mathrm{M}$, which is sufficient for detecting the 
nickel ions in drinking water (maximum allowable concentration is $1.7 \mu \mathrm{M}(0.2 \mathrm{mg} / \mathrm{l}))$.

\section{Disclosures}

All authors declare that there is no conflict of interests in this paper.

\section{Acknowledgements}

The study of ion-sensitive indicator synthesis conditions was supported by the Russian Foundation for Basic Research (grant No. 16-33-60100_mol_a_dk). The studies of luminescence characteristics of sensitive structures in the presence of analyte were supported by the Russian Scientific Foundation (project No. 14-5000034). The study of absorption spectra changes under the effect of different analyte concentrations was carried out at the expense of the Project ISGZ 0262-2014-0009. 